\title{
Chagas Disease Infection Prevalence and Vector Exposure in a High-Risk Population of Texas Hunters
}

\author{
Sarah M. Gunter, ${ }^{1 \star}$ Shannon E. Ronca, ${ }^{1}$ Micaela Sandoval, ${ }^{2}$ Kimberly Coffman, ${ }^{2}$ Lauren Leining, ${ }^{1,2}$ Rodion Gorchakov, ${ }^{1}$ \\ Kristy O. Murray, ${ }^{1}$ and Melissa S. Nolan ${ }^{1,3}$ \\ ${ }^{1}$ Section of Pediatric Tropical Medicine, Baylor College of Medicine and Texas Children's Hospital, Houston, Texas; ${ }^{2}$ The University of Texas Health \\ Science Center, School of Public Health, Houston, Texas; ${ }^{3}$ The University of South Carolina, Arnold School of Public Health, \\ Greenville, South Carolina
}

\begin{abstract}
Chagas disease, caused by the vector-borne parasite Trypanosoma cruzi, remains one of the most significant neglected tropical diseases affecting the Americas. Identifying high-risk populations is important for understanding Chagas disease transmission and directing public health resources. We recently hypothesized that Texas hunters may be at an elevated risk for contracting Chagas disease because of opportunities for vector exposure and contact with blood of infected reservoirs. To assess their unique exposure risks, we conducted a statewide screening program of Texas hunters. A total of 885 study participants were interviewed and tested for $T$. cruzi infection; 18 screened positive on a rapid, point-of-care test; however, none were found positive through confirmatory testing. We did find a high prevalence of reported direct contact with wildlife blood as well as triatomine and other arthropod disease vectors. This large-scale screening program represents a novel approach to better understand the vector-borne disease risk in this unique population.
\end{abstract}

Chagas disease, caused by the protozoan parasite Trypanosoma cruzi, remains one of the most significant neglected tropical diseases affecting the Americas. It is estimated that six to eight million people are infected with this parasite worldwide, with 238,091 infected individuals living in the United States. ${ }^{1-4}$ This disease causes progressive cardiac damage in about $30 \%$ of infected people, resulting in significant morbidity and mortality. ${ }^{5}$

The initial stages of Chagas disease are typically asymptomatic, making it difficult to detect cases early when treatment is more effective. ${ }^{6}$ Symptomatic cardiac disease does not usually develop until decades after infection. Therefore, identifying high-risk populations is important for understanding Chagas disease transmission and directing public health resources. We recently hypothesized that Texas hunters may be at an elevated risk for contracting Chagas disease. ${ }^{7-11}$ Hunters spend an extended amount of time outdoors in areas where sylvatic transmission of Chagas is likely, tend to stay overnight in substandard structures, and have frequent contact with potential mammalian reservoirs. ${ }^{7}$ All of these activities could place this population at an elevated risk for contracting Chagas disease. To assess their risk of exposure to $T$. cruzi and the triatomine vector, known locally as "kissing bugs" or "conenose bugs" (Triatoma spp.), we initiated a statewide screening program in Texas.

From August 2016 to May 2018, we invited study participants with a history of hunting in the state of Texas to participate in our Chagas screening program. Recruitment was conducted at public hunting areas, community events, and hunting expositions across the state. Working in collaboration with Texas Parks and Wildlife, we recruited participants from deer and feral hog hunts open to the public in 10 wildlife management areas, state parks, or the state's national areas. We also recruited from

\footnotetext{
*Address correspondence to Sarah M. Gunter, Section of Pediatric Tropical Medicine, Baylor College of Medicine and Texas Children's Hospital, 1102 Bates Ave., Suite 330.1, Houston, TX 77030. E-mail: sm22@bcm.edu
}

nine hunting expositions and seven community events (Figure 1A).

For each study participant who provided informed consent or had a parent or legal guardian who provided consent if younger than 18 years, we obtained a blood sample via venipuncture or finger stick and conducted an interview to obtain demographic information, hunting history, and a history of vector exposures. At the time of enrollment, the Chagas StatPak rapid assay (Chembio, Medford, NY) was used as a pointof-care test to obtain a preliminary result. If the participant allowed a full blood sample to be taken, an additional Hemagen Chagas Kit EIA (Hemagen Diagnostics, Columbia, MD) was later performed in our laboratory at Baylor College of Medicine. Specimens determined to be positive by Chagas Stat-Pak or Hemagen Chagas EIA were sent to the CDC, Division of Parasitic Diseases and Malaria, Parasitic Disease Reference Diagnostic Laboratory for confirmatory testing by Chagatest ELISA (Wiener Lab, Rossario, Argentina). All assays used in this study are commercially available and have been used previously in epidemiologic studies of Chagas disease. ${ }^{12,13}$ This study was approved by the Institutional Review Board at Baylor College of Medicine ( $\mathrm{H}-35471)$.

A total of 885 individuals, self-reported as hunters, participated in this study, representing 409 unique residential zip codes (Figure 1B). The cohort was predominately male (81\%), and white (95.6\%), non-Hispanic (86\%), with an average age of 48 years (Table 1). We believe this cohort is demographically representative of the 1.1 million Texans who hold a hunting license in the state, which comprises $87.6 \%$ men and $85 \%$ Caucasians, with the majority aged between 35 and 55 years $(53 \%) .^{14}$

Of the hunters who participated in the screening, we obtained surveys from 855 (96.6\%) participants. Our cohort reported an average of 31 years hunting (range, 1-75 years), with the majority of participants typically taking 2- to 3-daylong hunting trips (512/844). Of those who reported staying overnight for a hunting trip, the majority reported sleeping in a cabin (392/683), whereas others reported staying in tents $(236 / 683)$ or campers (243/683). Of concern, 78 participants 
A

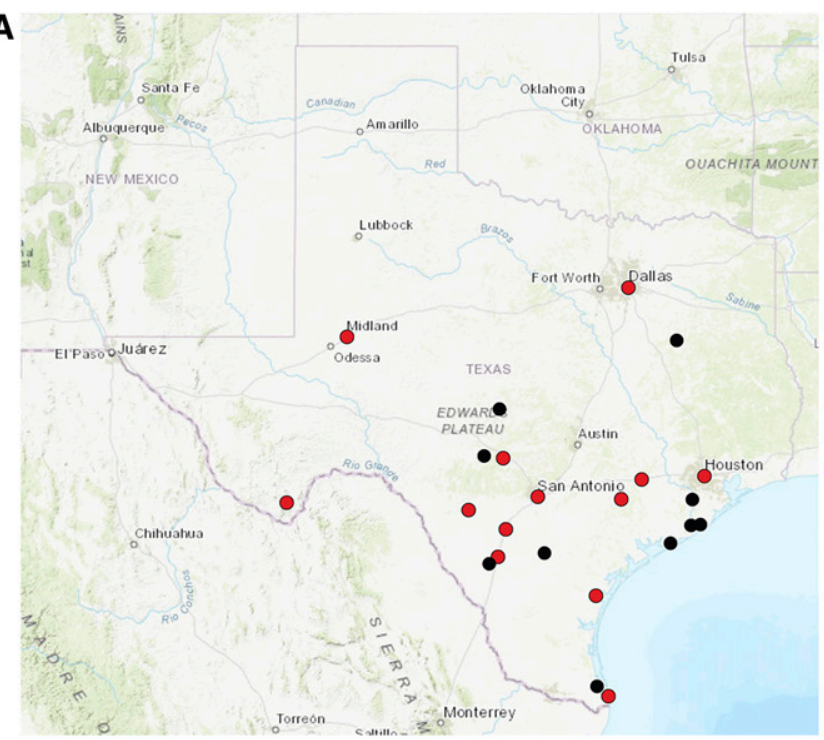

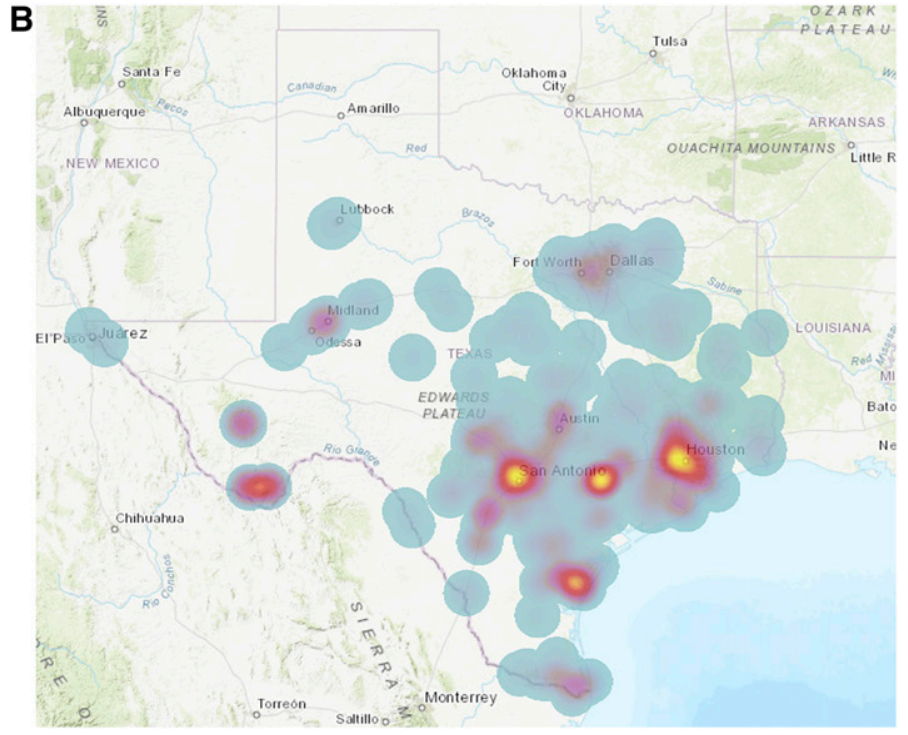

FIGURE 1. Participant recruitment. Map (A) depicts the 23 locations where recruitment events were held for this study (some locations had multiple events). Black dots represent a public hunting recruitment was conducted. Red dots represent a hunting expo or community event where recruitment was conducted. Map (B) depicts a heat map of residential zip codes reported by hunters enrolled in this study. This figure appears in color at www.aitmh.org.

reported sleeping outdoors with no shelter, elevating their risk of exposure to triatomines because of nocturnal feeding and $\mathrm{CO}_{2}$-seeking behaviors. ${ }^{2}$ In addition, almost half of these hunters (297/601) reported seeing insects where they slept while hunting. Although they did not identify triatomines directly, the evidence of insect infestations in their lodging suggests the potential for triatomines to infiltrate the structures (Table 2).

The majority of our cohort reported hunting white-tailed deer (Odocoileus virginianus; 799/855). In addition, participants reported hunting feral hogs (Sus scrofa; 632/855), small mammals (242/855), birds (447/855), fish (451/855), opossums (Didelphimorphia; 33/855), and armadillos (Dasypodidae; 32/855). Almost all reported field-dressing or processing the animals themselves (746/855), with a concerning majority (432/652) of the cohort reporting rarely or never using gloves when field-dressing, which allows for direct contact with blood and organs. We believe this lack of barrier protection use is concerning, as 23 mammalian

TABLE 1

Demographics of hunter participants, $n=885$

\begin{tabular}{lrr}
\hline & $\mathrm{N}$ & $\%$ \\
\hline Gender & & \\
Male & 714 & 81 \\
$\quad$ Female & 171 & 19 \\
Age (years) & & \\
$0-18$ & 26 & 3 \\
$19-40$ & 256 & 29 \\
$41-60$ & 372 & 42 \\
$60+$ & 224 & 25 \\
Not listed & 7 & 1 \\
Race & & \\
White & 846 & 95.6 \\
Asian & 3 & 1 \\
Black & 10 & 3 \\
Other/not listed & 26 & 14 \\
Ethnicity & & 1 \\
Hispanic & 123 & \\
\hline
\end{tabular}

species serve as reservoirs for Chagas disease in Texas, including white-tailed deer, feral hogs, opossums, and armadillos. 9,15,16 Without proper protection during the field-dressing process, there is a potential for blood-borne

TABLE 2

Hunting practices and vector exposures

\begin{tabular}{|c|c|}
\hline \multicolumn{2}{|l|}{ Hunting practices } \\
\hline Average hunting years (range) & $31(1-75)$ \\
\hline \multicolumn{2}{|l|}{ Average hunting trip, $n /$ total } \\
\hline 1 day & $188 / 844$ \\
\hline $2-3$ days & $512 / 844$ \\
\hline $1+$ week & $145 / 844$ \\
\hline Use a hunting structure & $694 / 854$ \\
\hline Box stand & $495 / 694$ \\
\hline Tree stand & $288 / 694$ \\
\hline Ground blind & $430 / 694$ \\
\hline Stay overnight while hunting & $683 / 855$ \\
\hline Open air & $78 / 683$ \\
\hline Hotel & $26 / 683$ \\
\hline Cabin & $392 / 683$ \\
\hline Tent & $236 / 683$ \\
\hline Camper & $243 / 683$ \\
\hline Other & $41 / 683$ \\
\hline See insects where you sleep & $297 / 601$ \\
\hline Field-dress animal & $746 / 855$ \\
\hline $\begin{array}{l}\text { Often or always wear gloves while field- } \\
\text { dressing }\end{array}$ & $220 / 746$ \\
\hline \multicolumn{2}{|l|}{ Vector exposure } \\
\hline Seen triatomines "kissing bugs" & $540 / 839$ \\
\hline Inside home & $83 / 540$ \\
\hline Outside home & $284 / 540$ \\
\hline While hunting & $233 / 540$ \\
\hline Bitten by triatomines & $32 / 855$ \\
\hline \multicolumn{2}{|l|}{ Bitten by mosquitos while hunting } \\
\hline Often/always & $578 / 855$ \\
\hline \multicolumn{2}{|l|}{ Bitten by ticks while hunting } \\
\hline Often/always & $118 / 855$ \\
\hline \multicolumn{2}{|l|}{ Insect repellent use while hunting } \\
\hline Never/rarely & $495 / 855$ \\
\hline
\end{tabular}


transmission if the hunter has any cuts or abrasions on the hand (Table 2).

When shown pictures of triatomines and resin-embedded Triatoma gerstaeckeri and Triatoma sanguisuga as visual aids, the majority of hunters reported having seen triatomines before (549/851). The hunters reported predominately seeing them outside their residential home and hunting lodge. Of concern, 83 participants from the cohort reported seeing them in their home, indicating the potential for domestic infestation. From our hunting cohort, 32 participants reported a triatomine bite history. In addition to triatomine exposure, this cohort reported a high prevalence of frequent mosquito (578/855) and tick (118/855) bites while hunting. Unfortunately, insect repellant use was not common among this cohort, with most participants (495/855) reporting rarely or never applying repellent while hunting. With such frequent exposure to these disease vectors, it is important to educate hunters and other outdoor enthusiasts to apply appropriate repellents to prevent vector-borne disease transmission (Table 2).

Among all participants, 18 had positive results for T. cruzi infection on the initial rapid test (2\%). From our cohort, $71 \%$ (624/885) allowed us to take a full blood sample for additional testing, including all 18 participants who initially screened positive. The Hemagen EIA indicated one positive and one indeterminate result of the 624 tested. None of the rapid test-positive individuals were also positive by Hemagen EIA. All 20 samples of the positive and indeterminate serum specimens ( 18 positive by Stat-Pak, one positive by EIA, and one indeterminate by EIA) were sent to CDC for confirmatory Weiner EIA. CDC confirmatory testing found none of the 20 samples positive. The current guidelines for diagnosis of Chagas disease requires two or more positive tests to confirm a case; therefore, we believe these individuals represent falsepositive results, which is expected considering the reported specificity of Chagas Stat-Pak is $97 \% .^{17,18}$

Although we likely did not find evidence of any cases of Chagas disease within this cohort of Texas hunters, we did find evidence of vector exposure and the potential for transmission. We also identified a gap in knowledge about Chagas disease, with only $40 \%$ (347/855) of participants reporting having heard of the disease before enrollment in the study. Most hunters reported staying overnight for their hunting trip, often in substandard or insect-infested lodging. Almost all hunters reported field-dressing wildlife carcasses, most of which are known reservoirs for $T$. cruzi, and doing so without gloves, introducing the potential for blood-borne transmission. Finally, a high proportion of this cohort reported having seen triatomines and some reported previous bites by the vector, indicating hunters are in areas of active sylvatic transmission cycles, and not using appropriate insect repellants.

This study does have some noteworthy limitations. The data collected from these surveys were self-reported and, as such, subject to response bias. When asking participants about triatomine exposure, we cannot rule out that they are misidentifying a "look-alike" insect. We believe that by showing participants several examples of local species in pictures and resin blocks, we have reduced the possibility of misclassification. We cannot rule out the potential for a falsenegative diagnosis in this cohort. We believe our testing algorithm was designed to limit the probability of this occurring, as our initial diagnostic, Chagas Stat-Pak, has previously been found to have high sensitivity, and we encouraged individuals to allow for a second diagnostic regardless of the initial testing results. ${ }^{12}$ Although we have followed the standard serologic diagnostic protocol, the low potential for misdiagnosis exists. Finally, our sample size may be too small to accurately detect the prevalence of Chagas disease in this population, as only one in 6,500 blood donors in the state is identified as Chagas positive. ${ }^{19,20}$ Larger surveillance efforts are necessary to truly determine the prevalence of disease in this population.

This large-scale screening program represents a novel approach to better understand Chagas disease transmission and vector exposure in this high-risk population in the Southern United States. The present study identified a risk of triatomine and other vector exposures during activities associated with hunting. Most significantly, this study illustrates the significant knowledge gap surrounding Chagas disease and the steps hunters can take to prevent becoming infected. We believe these findings highlight the importance of enhanced public health campaigns targeting unique populations, such as hunters, who may be at an increased exposure risk in Texas.

Received April 23, 2019. Accepted for publication October 10, 2019. Published online December 23, 2019.

Acknowledgments: We would like to thank Texas Parks and Wildlife for their help in facilitating access to public hunting events for this study and the study participants for their time.

Financial support: This study was funded by a grant from the National Institutes of Health (NIH R21-Al112647-02).

Authors' addresses: Sarah Gunter, Shannon E. Ronca, Rodion Gorchakov, and Kristy O. Murray, Section of Pediatric Tropical Medicine, Baylor College of Medicine and Texas Children's Hospital, Houston, TX, E-mails: sm22@bcm.edu, sronca@bcm.edu, rodion.gorchakov@bcm.edu, and kmurray@bcm.edu. Micaela Sandoval and Kimberly Coffman, The University of Texas Health Science Center, School of Public Health, Houston, TX, E-mails: micaela.n.sandoval@ uth.tmc.edu and kdjones@medicine.tamhsc.edu. Lauren Leining, Section of Pediatric Tropical Medicine, Baylor College of Medicine and Texas Children's Hospital, Houston, TX, and The University of Texas Health Science Center, School of Public Health, Houston, TX, E-mails: lauren.leining@bcm.edu or lauren.m.leining@uth.tmc.edu. Melissa S. Nolan, Baylor College of Medicine and Texas Children's Hospital, Section of Pediatric Tropical Medicine, Houston, TX, and The University of South Carolina, Amold School of Public Health, Greenville, SC, E-mails: msnolan@ mailbox.sc.edu or mnolan@bcm.edu.

\section{REFERENCES}

1. WHO, 2018. Chagas Disease (American Trypanosomiasis)Epidmeiology. Available at: https://www.who.int/chagas/ epidemiology/en/. Accessed March 12, 2019.

2. Bern C, Kjos S, Yabsley MJ, Montgomery SP, 2011. Trypanosoma cruzi and Chagas' disease in the United States. Clin Microbiol Rev 24: 655-681.

3. Bern C, Montgomery SP, 2009. An estimate of the burden of Chagas disease in the United States. Clin Infect Dis 49: e52-e54.

4. Manne-Goehler J, Umeh CA, Montgomery SP, Wirtz VJ, 2016. Estimating the burden of Chagas disease in the United States. PLoS Negl Trop Dis 10: e0005033.

5. Sabino EC et al.; National Heart, Lung, and Blood Institute Retrovirus Epidemiology Donor Study-II (REDS-II), International Component, 2013. Ten-year incidence of Chagas cardiomyopathy among asymptomatic Trypanosoma cruzi-seropositive former blood donors. Circulation 127: 1105-1115.

6. Morillo CA, Marin-Neto JA, Avezum A, 2016. Benznidazole for chronic Chagas' cardiomyopathy. N Engl J Med 374: 189-190. 
7. Garcia MN, Murphy SK, Gross A, Wagner J, Murray KO, 2015. Knowledge, attitudes, and practices of Texas hunters: a potentially high-risk population for exposure to the parasite that causes Chagas disease. Parasit Vectors 8: 197.

8. Gunter SM et al., 2017. Likely autochthonous transmission of Trypanosoma cruzi to humans, South Central Texas, USA. Emerg Infect Dis 23: 500-503.

9. Gunter SM, Cordray C, Gorchakov R, Du I, Dittmar B, Brown EL, Murray KO, Nolan MS, 2018. Identification of white-tailed deer (Odocoileus virginianus) as a novel reservoir species for Trypanosoma cruzi in Texas, USA. J Wildl Dis 54: 814-818.

10. Garcia MN, Aguilar D, Gorchakov R, Rossmann SN, Montgomery SP, Rivera H, Woc-Colburn L, Hotez PJ, Murray KO, 2015. Evidence of autochthonous Chagas disease in southeastern Texas. Am J Trop Med Hyg 92: 325-330.

11. Garcia MN, Hotez PJ, Murray KO, 2014. Potential novel risk factors for autochthonous and sylvatic transmission of human Chagas disease in the United States. Parasit Vectors 7: 311.

12. Afonso AM, Ebell MH, Tarleton RL, 2012. A systematic review of high quality diagnostic tests for Chagas disease. PLoS Negl Trop Dis 6: e1881.

13. Meymandi S, Hernandez S, Park S, Sanchez DR, Forsyth C, 2018. Treatment of Chagas disease in the United States. Curr Treat Options Infect Dis 10: 373-388.

14. Wilder HK, Wozniak E, Huddleston E, Tata SR, Fitzkee NC, Lopez JE, 2015. Case report: a retrospective serological analysis indicating human exposure to tick-borne relapsing fever spirochetes in Texas. PLoS Negl Trop Dis 9: e0003617.

15. Gunter SM, Brown EL, Gorchakov R, Murray KO, Garcia MN, 2017. Sylvatic transmission of Trypanosoma cruzi among domestic and wildlife reservoirs in Texas, USA: a review of the historical literature. Zoonoses Public Health 64: 313-327.

16. Comeaux JM, Curtis-Robles R, Lewis BC, Cummings $\mathrm{KJ}$, Mesenbrink BT, Leland BR, Bodenchuk MJ, Hamer SA, 2016. Survey of feral swine (Sus scrofa) infection with the agent of Chagas disease (Trypanosoma cruzi) in Texas, 2013-14. J Wildl Dis 52: 627-630.

17. CHEMIBIO, 2017. Chagas Stat-Pak Assay. Available at: http:// chembio.com/wp-content/uploads/2017/02/10-6192-0-ChagasSTAT-PAK-Assay-IFU-Rev-5.pdf. Accessed March 27, 2019.

18. CDC, 2014. Parasites - American Trypanosomiasis (Also Known as Chagas Disease). Available at: https://www.cdc.gov/parasites/ chagas/health_professionals/dx.html. Accessed March 27, 2019.

19. Garcia MN et al., 2016. Trypanosoma cruzi screening in Texas blood donors, 2008-2012. Epidemiol Infect 144: 1010-1013.

20. Webber BJ, Pawlak MT, Valtier S, Daniels CC, Tully CC, Wozniak EJ, Roachell WD, Sanchez FX, Blasi AA, Cropper TL, 2017. Prevalence and seroprevalence of Trypanosoma cruzi infection in a military population in Texas. Am J Trop Med Hyg 97: 1477-1481. 